\title{
Electric field and temperature-induced removal of moisture in nanoporous organosilicate films
}

\author{
N. Biswas ${ }^{\mathrm{a})}$ \\ Nano Tech Center, Texas Tech University, Lubbock, Texas 79409 \\ J. A. Lubguban and S. Gangopadhyay ${ }^{\text {) }}$ \\ Department of Electrical and Computer Engineering, University of Missouri, Columbia, Missouri 65211
}

(Received 12 January 2004; accepted 12 April 2004; published online 7 May 2004)

\begin{abstract}
The effects of bias-temperature-stress (BTS) or simply temperature-stress (TS) on nanoporous low- $k$ methylsilsesquioxane films are studied. Initially, the as-given and $\mathrm{O}_{2}$ ashed/etched films exhibit physical adsorption of moisture as revealed from the electrical behavior of the samples after 15 days. The temperature stressing at $170{ }^{\circ} \mathrm{C}$ volatilized the adsorbed water but is unable to remove chemisorb and hydrophillic $\mathrm{Si}-\mathrm{OH}$ groups. As a result, the TS films remain susceptible to moisture. BTS at $170^{\circ} \mathrm{C}$ also removes adsorbed water. More important, the surfaces under the metal-insulator structure were dehydroxylated by breaking the chemisorb $\mathrm{Si}-\mathrm{OH}$ group facilitating the formation of siloxane bonds that prevents adsorption of moisture even after 60 days. (C) 2004 American Institute
\end{abstract} of Physics. [DOI: 10.1063/1.1757019]

As device dimensions shrink, the struggle to find ultralow dielectric constant $(k)$ materials for future generation integrated circuits (ICs) continues unabated. Although many low- $k$ materials in the range of $2.0-4.0^{1-5}$ have been studied, integration problems and issues like moisture adsorption brought numerous reliability concerns. Methylsilsesquixane (MSQ) is an attractive candidate for replacement of $\mathrm{SiO}_{2}$ in future generation ICs because of its inherent low- $k$ (2.8) and good thermal stability. The introduction of nanopores to MSQ provides extendibility of dielectric constant to values lower than 2.0. However, the dielectric properties of this nanoporous material are seriously degraded during photoresist removal - an important microelectronic fabrication step that involves $\mathrm{O}_{2}$ ashing/etching. ${ }^{6,7}$ This process also removes the hydrophobic $\mathrm{CH}_{x}$ groups from exposed surfaces (pore surface as well) of the film and allowing the formation of silanol $(\mathrm{Si}-\mathrm{OH})$ groups. As a result, the hydrophillic $\mathrm{Si}-\mathrm{OH}$ enhances physical adsorption of moisture upon air exposure and increases the dielectric constant. This causes device reliability problems. Device instabilities could occur causing an increase in leakage currents that may lead to breakdown of the dielectric film.

Current work focuses on studying the effects of biastemperature-stress (BTS) or temperature-stress (TS) of nanoporous MSQ. BTS has been a very effective method to detect device instabilities like the presence of mobile ions or diffusion of metal ions in the dielectric. The time-dependent moisture adsorption and thermal stability of as-given and $\mathrm{O}_{2}$ ashed/etched MSQ films are also investigated. This study will attempt to explain the susceptibility of MSQ films to moisture after BTS or TS.

The nanoporous MSQ films studied are courtesy of Tokyo Electron America (TEL). The as-given and $\mathrm{O}_{2}$ ashed/

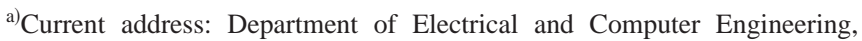
North Carolina State University, Raleigh, NC 27695.

b) Author to whom correspondence should be addressed; electronic mail: gangopadhyays@missouri.edu
}

etched MSQ for electrical measurements have thicknesses of 390 and $280 \mathrm{~nm}$, respectively. To fabricate metalinsulator-semiconductor (MIS) devices, metal dots of titanium with an area $4.9 \times 10^{-4} \mathrm{~cm}^{2}$ were sputter-deposited. The capacitance-voltage $(C-V)$ measurements were carried out on HP4284 LCR meter while current-voltage measurements were carried out on Keithly 2400 Sourcemeter. To avoid errors due to series resistance effects, ${ }^{8} C-V$ curves were measured at a lower frequency $(1 \mathrm{kHz})$. The accumulation capacitance of the $C-V$ curves was used to calculate the dielectric constants of the films. To confirm the accuracy in the dielectric constants, the capacitance of the same films deposited on heavily doped silicon was also measured. BTS experiments were performed at $170{ }^{\circ} \mathrm{C}$ at an electric field of -0.4 to $-0.7 \mathrm{MV} / \mathrm{cm}$ and at biases pertaining to the accumulation region. The samples were subjected to bias at $170{ }^{\circ} \mathrm{C}$ for $20 \mathrm{~min}$. The samples were then cooled and brought down to room temperature with the bias on. Temperature stress was also done at $170^{\circ} \mathrm{C}$ for $20 \mathrm{~min}$. $C-V$ scans were carried out on MIS structure immediately after BTS and TS.

Figure 1 shows $C-V$ plots of film A (as-given MSQ) and D (ashed/etched MSQ) measured within $48 \mathrm{~h}$ after receiving the samples from TEL in a vacuum-sealed container. This time will be referred to as $t=0$ henceforth. The dielectric constants measured were 2.25 for sample A and 3.0 for sample D. These values were in agreement with those obtained from same films deposited on heavily doped silicon and averaged over at least 7 MIS structures. The higher value of $k$ for film $\mathrm{D}$ is due to the removal of $\mathrm{CH}_{x}$ groups and formation of $\mathrm{Si}-\mathrm{OH}$ groups upon $\mathrm{O}_{2}$ ashing/etching. The samples were then left at room temperature for 15 days to test its stability in air exposure. The same MIS structures were re-measured after 15 days. Curves A1 and D1 in Figs. 2(a) and 2(b), respectively, represent the resulting $C-V$ plots. The curves show an increase in accumulation capacitances and a shift toward positive biases relative to the virgin samples [solid lines in Figs. 2(a) and 2(b)]. The increase in 


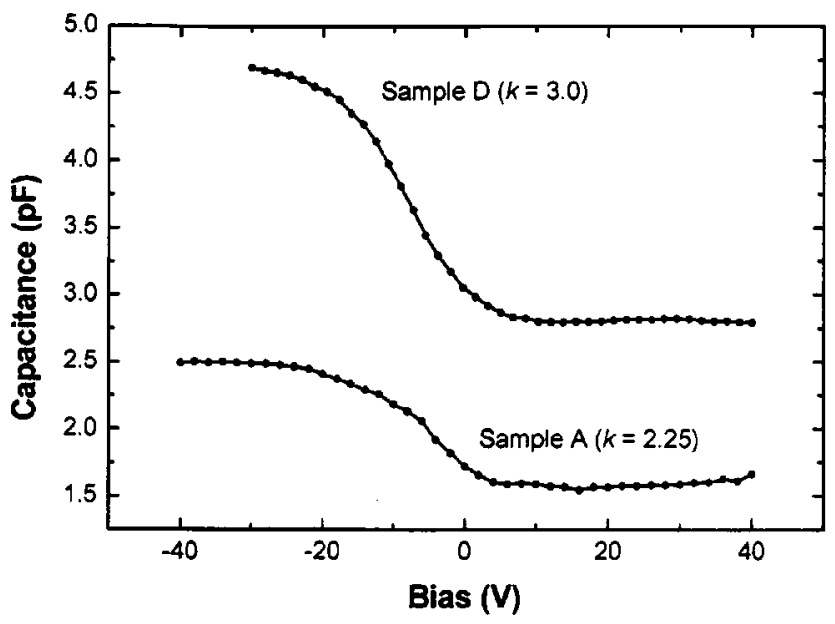

FIG. 1. $C-V$ curves of virgin sample A and sample D.

accumulation capacitance will increase $k$. The increase in accumulation capacitance for sample $\mathrm{D}$ is the result of physically adsorbed water upon air exposure. The nonvicinal silanol groups like isolated $\mathrm{Si}-\mathrm{OH}$ formed after $\mathrm{O}_{2}$ ashing/ etching acts as sites of adsorption through hydrogen bonding. It is well known that the addition of polar molecules like water and silanols increase the dielectric constant. The accumulation capacitance increase for sample A (curve A1) may also be due to the above-mentioned phenomenon. Though the surface of MSQ is terminated with hydrophobic $\mathrm{CH}_{x}$, isolated silanols may be present from incomplete aging or
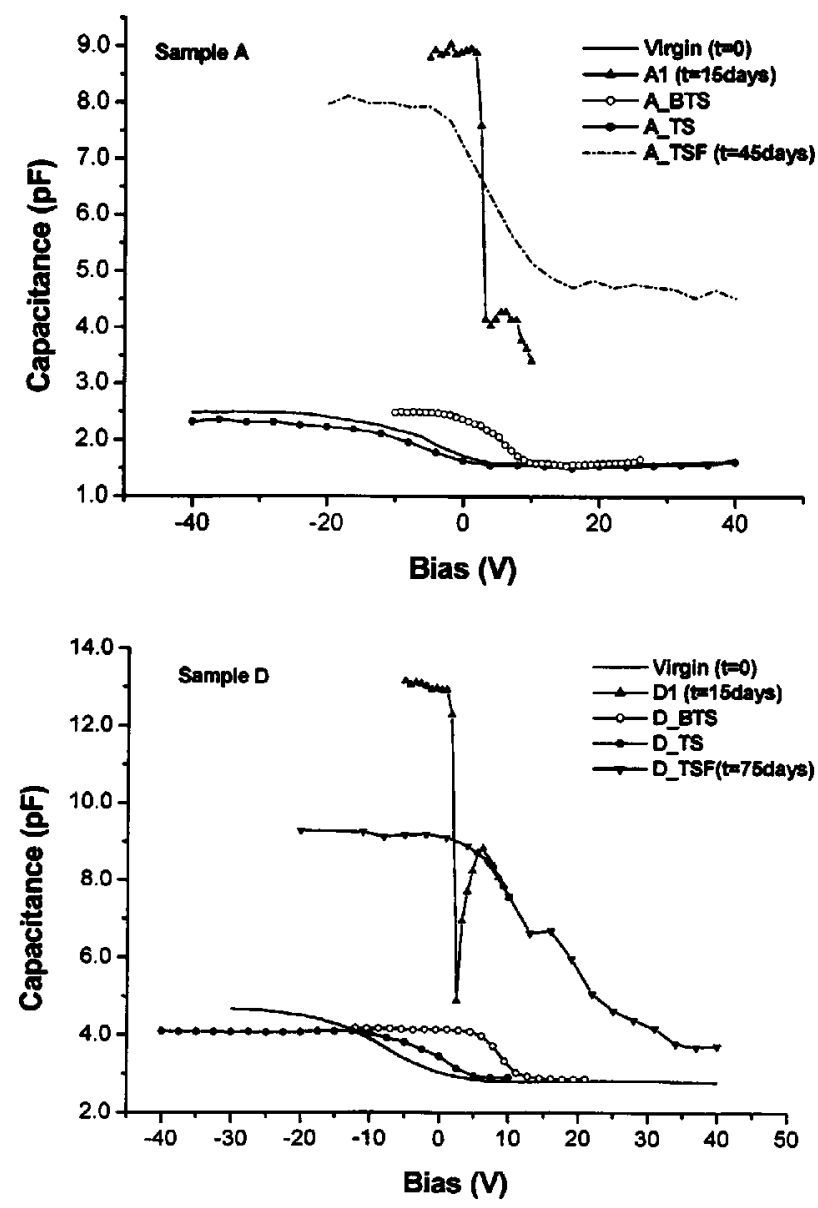

FIG. 2. BTS/TS history of sample A [Fig. 2(a)] and sample D [Fig. 2(b)]. Downloaded 12 Aug 2010 to 128.206.162.204. Redistribution subject to A

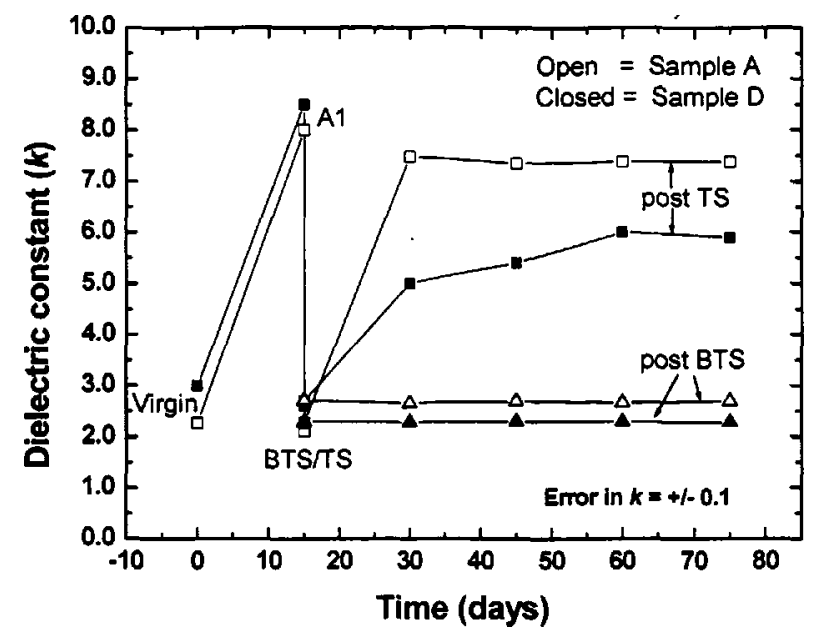

FIG. 3. Summary of change of dielectric constant with time before and after BTS/TS.

curing at a temperature not sufficient to remove surface silanols.

The two sets of samples were then subjected to TS or BTS. The resulting curves for samples $\mathrm{A}$ and $\mathrm{D}$ are also shown in Figs. 2(a) and 2(b). TS at $170{ }^{\circ} \mathrm{C}$ removes the physically adsorbed water in the films. In studies of amorphous silica, the hydrogen-bonded adsorbed water is easily removed at temperatures of $25-170{ }^{\circ} \mathrm{C}$. ${ }^{9}$ The removal of water by TS causes the accumulation capacitance to decrease and almost equal that of the virgin sample (curves A_TS and D_TS). The curves also shift back toward the curves for the virgin samples. The BTS samples (curves A_BTS and D_BTS) show almost the same decrease in capacitance. However, the shifts are toward positive biases-in opposite direction of A_TS and D_TS relative to A1 and D1, respectively.

To test the susceptibility to atmospheric moisture, $C-V$ scans were repeated over the BTS and TS gates after air exposure for 15 days. Within error of measurements, the new $C-V$ curves for BTS gates did not show significant change when compared to the as-treated A_BTS and D_BTS curves, respectively. The new curves are not plotted in Fig. 2 for clarity. This result indicates that BTS treatment prevents moisture re-adsorption in the MIS structures. The new $C-V$ curves for the TS gates after 15 days show rise in accumulation capacitance (not shown in Fig. 2) with $k=7.48$ for sample A and $k=5$ for sample $\mathrm{D}$. The $C-V$ curves of both TS samples after a time of 75 days are shown in Figs. 2(a) and 2(b) (curves A_TSF and D_TSF), respectively. The curves also show an increase in accumulation capacitance and shift of $C-V$ curves toward positive biases relative to as-treated TS curves. This trend correlates well with moisture re-adsorption after air exposure.

Figure 3 is a plot of the change in dielectric constants with time in days and also with BTS or TS. After TS, the dielectric constant for samples A and D increases with time and saturates after 30 days of air exposure. The dielectric constant of both samples after BTS did not change at all even up to 60 days. This clearly shows that BTS prevents rehydration after being exposed to air while TS does not.

As stated earlier, the dehydration of samples after TS is due to the volatilization of physically adsorbed water at-
license or copyright; see http://apl.aip.org/about/rights and permissions 


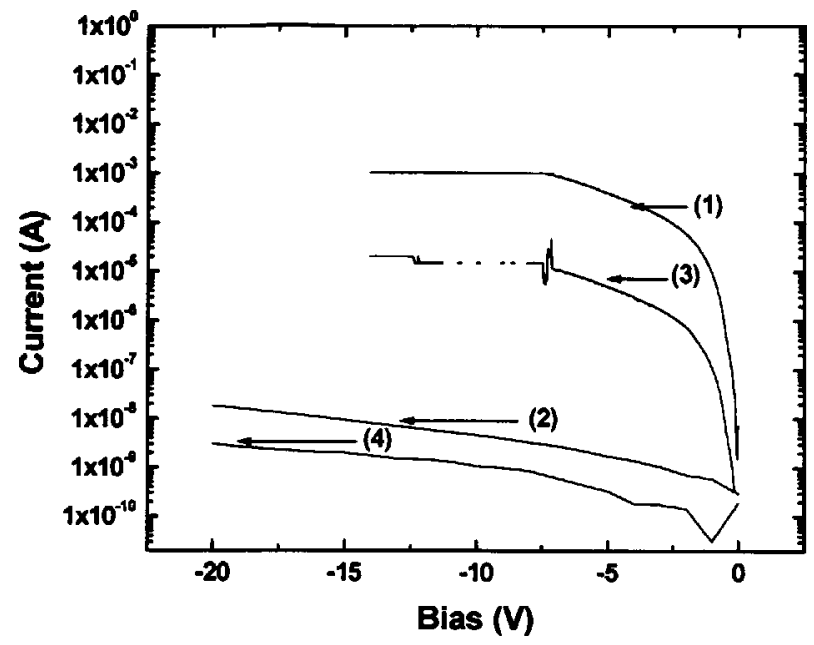

FIG. 4. Current-voltage history for sample D. (a) D1, (2) D_TS, (3) D_TSF, and (4) D_BTS.

tached to the chemisorb silanol groups. But the $170{ }^{\circ} \mathrm{C}$ temperature is not sufficient to dehydroxylate the surface from chemisorb silanols. Hence, the samples are rehydrated upon air exposure. Apparently, the additional electric field with temperature during BTS volatilized adsorb water and also enhanced dehyroxylation of chemisorb silanols under the MIS structures. The dehydroxylation involves the release of water on neighboring silanols leaving a siloxane surface that is essentially hydrophobic. Studies by Young and Bursh have clearly shown that water molecules are adsorbed only on the hydroxylated silica surface and not on siloxane surface. ${ }^{10,11}$ Hydration can still occur on siloxane oxygen sites next to a silanol site. The surfaces dehydrated up to $400-425^{\circ} \mathrm{C}$ were readily rehydrated. ${ }^{10,11}$ Above this temperature, rehydration becomes progressively slow. Agzamkhodzhaev et al. found that the more the silica surface is dehydrated, the longer the time for rehydration. ${ }^{12}$

Figure 4 shows the current-voltage $(I-V)$ history of sample D. Curve 1, 2, and 3 represent the $I-V$ curves corresponding to the $C-V$ curves D1, D_TS, and D_TSF, respectively, of Fig. 2(b). The high leakage represented by curve 1 is due to the formation of $\mathrm{Si}-\mathrm{OH}$ groups in the MSQ film during $\mathrm{O}_{2}$ ashing/etching and physical adsorption of water upon air exposure. Curve 2 represents expulsion of adsorbed water and hence lowered leakage. Upon air exposure, moisture is re-adsorbed and the leakage current has increased (curve 3) by almost four orders of magnitude. Curve 4 represents the $I-V$ curve for BTS metal gate, of curve D_BTS in Fig. 2(b). The $I-V$ curve for this metal gate was monitored over time along with the $C-V$ curves in Fig. 2(b). No apparent change in the leakage current was recorded. The subsequent $I-V$ curves are not shown in Fig. 4.

In conclusion the effects of TS and BTS on nanoporous MSQ films have been investigated. Both as-given and $\mathrm{O}_{2}$ ashed/etched MSQ were found susceptible to atmospheric moisture adsorption. The temperature stressing removes the adsorbed water. However, the presence of $\mathrm{Si}-\mathrm{OH}$ bonds sustains the samples receptiveness to atmospheric moisture. After BTS of both samples, moisture adsorption has been prevented under the MIS structure due to removal of chemisorb silanols and formation of siloxanes on the surface.

The authors would like to acknowledge Dorel Toma of TEL for providing the samples, and SRC and NSF for funding this research.

${ }^{1}$ M. G. Albrecht and C. Blanchette, J. Electrochem. Soc. 145, 4019 (1998).

${ }^{2}$ S. M. Rossnagel, Proceedings of the VLSI Multilevel Interconnection Conference (VMIC), 1995.

${ }^{3}$ M. J. Loboda, C. M. Grove, and R. F. Schneider, J. Electrochem. Soc. 145, 2861 (1998)

${ }^{4}$ P. T. Liu, T. C. Chang, Y. L. Yang, Y. F. Cheng, and S. M. Sze, IEEE Trans. Electron Devices 47, 1733 (2000).

${ }^{5}$ A. Grill, V. Patel, K. L. Saenger, C. Jahnes, S. A. Cohen, A. G. Schrott, D. C. Edelstein, and J. R. Parasszczak, Mater. Res. Soc. Symp. Proc. 443, 155 (1996).

${ }^{6}$ P. T. Liu, T. C. Chang, Y. S. Mor, and S. M. Sze, Jpn. J. Appl. Phys., Part 1 38, 3482 (1999).

${ }^{7}$ P. T. Liu, T. C. Chang, S. M. Sze, F. M. Pan, Y. J. Mei, W. F. Wu, M. S. Tsai, B. T. Dai, C. Y. Chang, F. Y. Shih, and H. D. Huang, Thin Solid Films 332, 345 (1998).

${ }^{8}$ E. H. Nicollian and J. R. Brews, MOS Physics and Technology (Wiley, New York, 1982).

${ }^{9}$ R. K. Iler, The Chemistry of Silica (Wiley, New York, 1979), p. 625.

${ }^{10}$ G. J. Young, J. Colloid Sci. 13, 67 (1958).

${ }^{11}$ G. J. Young and T. P. Bursch, J. Colloid Sci. 15, 361 (1960).

${ }^{12}$ A. A. Agzamkhodzhaev, L. T. Zhuravlev, A. Kiselev, and K. Y. Shengeliya, Kolloidn. Zh. 36, 1145 (1974). 